$03,07,10$

\title{
Распределение дислокаций несоответствия и упругих механических напряжений в метаморфных буферных слоях InAIAs разной конструкции
}

\author{
(С) Д.В. Побат, В.А. Соловьев, М.Ю. Чернов, С.В. Иванов \\ Физико-технический институт им. А.Ф. Иофрфе РАН, \\ Санкт-Петербург, Россия \\ E-mail: pobat.dima@gmail.com
}

Поступила в Редакцию 18 сентября 2020 г.

В окончательной редакции 18 сентября 2020 г.

Принята к публикации 19 сентября 2020 г.

\begin{abstract}
Проведен расчет равновесных распределений плотности дислокаций несоответствия $\rho(z)$ и упругих напряжений $\varepsilon(z)$ вдоль направления эпитаксиального роста метаморфного буферного слоя InAlAs/GaAs $(001)$ с высоким содержанием In (до $87 \mathrm{~mol} \%$ ) и разными профилями изменения состава: ступенчатым, линейным и корневым. Для расчетов был использован метод, основанный на итерационном поиске минимального значения полной энергии системы. Показано, что наиболее сильные отличия между разными конструкциями буферного слоя наблюдаются в характере распределений $\rho(z)$, а не $\varepsilon(z)$. В отличие от традиционных конструкций со ступенчатым и линейным градиентом состава, которые характеризуются достаточно однородным распределением дислокаций несоответствия, в буферном слое с корневым градиентом состава основная часть таких дислокаций сконцентрирована в нижней части слоя вблизи гетерограницы с подложкой GaAs, a иx плотность резко падает более чем на порядок величины по толщине слоя, достигая вблизи поверхности минимального из всех перечисленных конструкций значения. Несмотря на то, что в данной работе не учитывался важный эффект взаимодействия дислокаций между собой, проведенные расчеты позволили установить основные особенности распределений $\rho(z)$ и $\varepsilon(z)$ в различных метаморфных буферных слоях InAlAs, которые ранее наблюдались нами экспериментально. Таким образом, данный подход может быть эффективно использован при создании оптимальных конструкций приборных метаморфных гетероструктур.
\end{abstract}

Ключевые слова: дислокации несоответствия, упругие напряжения, метаморфные гетероструктуры, InAlAs/GaAs, большое рассогласование параметров решетки.

DOI: 10.21883/FTT.2021.01.50403.201

\section{1. Введение}

Использование полупроводниковых гетероструктур на основе твердых растворов $\operatorname{In}_{x}(\mathrm{Al}, \mathrm{Ga})_{1-x}$ As с высоким содержанием In $(x \geq 0.7)$ позволяет создавать высокоэффективные приборы современной электроники, включая транзисторы с высокой подвижностью электронов [1], биполярные транзисторы на гетеропереходах [2], а также полупроводниковые светодиоды и лазеры, излучающие в среднем инфракрасном диапазоне $(2-5 \mu \mathrm{m})$ [3-4]. Интересной является реализация подобных структур на подложках GaAs, которые отличаются высокой технологичностью и низкой стоимостью по сравнению с традиционно используемыми подложками $\mathrm{InSb}$ и $\mathrm{GaSb}$. Однако, сильное рассогласование параметров кристаллической решетки подложки $\left(a_{s}\right) \mathrm{GaAs}$ и активной области (a) $\Delta a / a>5 \%$ приводит к формированию большой плотности дислокаций несоответствия (ДН), a также прорастающих дислокаций (ПД), оказывающих негативное влияние на выходные параметры структур.

Одним из эффективных подходов для решения данной проблемы является применение метаморфного буферного слоя (МБС), представляющего собой слой твердого раствора переменного состава, в котором по мере его роста параметр решетки постепенно изменяется от $a_{s}$ до требуемой величины. Традиционно используются МБС со ступенчатым либо линейным профилем изменения состава [5], что связано с простотой реализации таких МБС при выращивании метаморфных структур методом молекулярно-пучковой эпитаксии (МПЭ) и возможностью применения комплексной теории релаксации упругих напряжений, разработанной Данстаном [6,7], для оценки и контроля плотности образующихся в структуре дислокаций и остаточных упругих механических напряжений. Отсутствие подобной теории для нелинейных МБС, наряду со сложностями технической реализации нелинейного профиля изменения состава, сдерживает их активное применение в метаморфных структурах, хотя было показано, что использование МБС $\operatorname{In}_{x} \mathrm{Al}_{1-x} \mathrm{As}$ с нелинейным градиентом состава и максимальным содержанием In в нем $x_{\max }>0.5$, вместо линейного или ступенчатого МБС, позволяет получить на порядок величины ниже плотность ПД $\left(\sim 10^{7} \mathrm{~cm}^{-2}\right)$, а также увеличить толщину так называемой бездислокационной (dislocation free) области $\left(d_{\text {free }}\right)$ [8,9]. Несмотря на отсутствие теории и малое количество опубликованных экспериментальных работ, для понимания процессов 
релаксации упругих напряжений и распределения ДН в МБС с нелинейным профилем изменения состава достаточно эффективным может оказаться численный подход [10].

Настоящая работа посвящена моделированию профилей распределения плотности ДН и упругих напряжений вдоль направления эпитаксиального роста МБС $\mathrm{In}_{x} \mathrm{Al}_{1-x} \mathrm{As}\left(x_{\max }=0.75-0.87\right)$ с линейным, ступенчатым и корневым градиентом изменения состава, а также сравнению полученных результатов моделирования с экспериментальными данными.

\section{2. Расчет упругих механических напряжений и плотности дислокаций несоответствия в МБС}

В настоящей работе для расчета равновесных профилей распределения упругих напряжений и плотности ДН в направлении роста исследуемых структур с разным типом МБС был использован численный метод, подробно изложенный в работе Bertoli и др. [10]. Этот метод (метод последовательных приближений) основан на итерационном поиске минимального значения полной энергии системы, которая включает в себя упругую энергию $\left(E_{\varepsilon}\right)$ и энергию ДН $\left(E_{d}\right)$. Далее приводится краткое описание алгоритма поиска минимума полной энергии системы с устранением некоторой неясности его изложения, а также неточностей в написании конечных формул, обнаруженных нами в работе [10].

МБС, суммарной толщиной $L$, разбивается на $N$ подслоев, каждый из которых обладает своим собственным набором параметров: толщиной подслоя $h_{i}$, параметром кристаллической решетки $a_{i}$ и модулями упругости $C_{k l}$ (см. таблицу). Учитывая, что точность расчетов повышается с уменьшением значений $h_{i}$, количество подслоев $N$ выбиралось таким, чтобы $h_{i}$ не превышали $5 \mathrm{~nm}$. Упругие напряжения в слое определяются в соответствии с выражением (1):

$$
\varepsilon_{i}=f_{i}+\sum_{m=1}^{i} b_{m}^{\prime} \rho_{m} h_{m}
$$

где $f_{i}=\left(a_{s}-a_{i}\right) / a_{i}-$ рассогласование параметров решеток подслоя $i$ и подложки, $b_{m}^{\prime}$ - проекция вектора Бюргерса на ось, совпадающую с направлением роста, $\rho_{i}$ - величина плотности ДН в подслое $i$.

Выражение для упругой энергии системы на единицу площади имеет вид

$$
E_{\varepsilon}=\sum_{i=1}^{N} \varepsilon_{i}^{2} Y_{i} h_{i}
$$

где $Y_{i}=C_{11 i}+C_{12 i}-2 C_{12 i}^{2} / C_{11 i}-$ модуль Юнга в направлении [001] [12]. Формула для расчета энергии
Параметры исследуемых в данной работе материалов [11]

\begin{tabular}{c|l|l|c|c}
\hline Материал & \multicolumn{1}{|c|}{$a, \mathrm{~nm}$} & $b, \mathrm{~nm}$ & $C_{11}, \mathrm{GPa}$ & $C_{12}, \mathrm{GPa}$ \\
\hline $\mathrm{GaAs}$ & 0.56534 & 0.400 & 118.4 & 53.7 \\
$\mathrm{AlAs}$ & 0.5611 & 0.400 & 120.2 & 57.0 \\
$\mathrm{InAs}$ & 0.60584 & 0.428 & 83.29 & 45.26
\end{tabular}

дислокаций на единицу площади может быть записана следующим образом:

$$
E_{d}=\sum_{i=1}^{N} \frac{G_{i} b_{i}^{2}\left(1-v_{i} \cos ^{2} \alpha\right)}{2 \pi\left(1-v_{i}\right)}\left[\ln \left(\frac{L-\sum_{k=1}^{i-1} h_{k}}{b_{i}}\right)+1\right] \rho_{i} h_{i}
$$

где $\alpha-$ угол между вектором Бюргерса и линией дислокации, $G_{i}=\left(C_{11 i}-C_{12 i}\right) / 2$ и $\nu_{i}=C_{12 i} /\left(C_{11 i}+C_{12 i}\right)$ модуль сдвига и коэффициент Пуассона, соответственно [12]. Как известно, в напряженных полупроводниковых гетероструктурах $\mathrm{A}^{3} \mathrm{~B}^{5}$ с типом кристаллической решетки цинковой обманки наиболее характерными ДН являются $60^{\circ}$ дислокации $[13,14]$. Поэтому в расчетах использовалось значение $\alpha=60^{\circ}$.

В качестве исходных данных расчета задаем начальные значения и для простоты принимаем $\rho_{i}$ в каждом подслое равным 0 , при этом $\varepsilon_{i}=f_{i}$, что соответствует полному отсутствию релаксации в структуре. Далее для каждого подслоя $i$, начиная с первого, определяем первое итерационное значение $\rho_{i}^{1}$. Для этого вводим в рассмотрение по три значения ,Плотности ДН“ для данного слоя $(i): \rho_{i}^{\text {up }}=\rho_{i}+\Delta \rho, \rho_{i}^{\text {const }}=\rho_{i}$, $\rho_{i}^{\text {down }}=\rho_{i}-\Delta \rho$ и для нескольких соседних с ним слоев $(i+1, i+2$ и т. д.). Например, в случае одновременного рассмотрения двух слоев $(i$ и $i+1)$ выражения для трех значений „плотности ДН“ в слое $i+1$ будут иметь вид: $\rho_{i+1}^{\text {up }}=\rho_{i+1}+\Delta \rho, \rho_{i+1}^{\text {const }}=\rho_{i+1}, \quad \rho_{i+1}^{\text {down }}=\rho_{i+1}-\Delta \rho$. Отметим, что перечисленные компоненты могут быть как положительными, так и отрицательными. При этом, положительное значение соответствует случаю упругих напряжений растяжения, а отрицательное - напряжениям сжатия. Затем в соответствии с выражениями (1-3) производится расчет полной энергии системы $E_{\varepsilon}+E_{d}$ для различных комбинаций значений „плотности ДН“ в рассматриваемых соседних слоях и выбирается та комбинация, которая соответствует минимальному значению величины $E_{\varepsilon}+E_{d}$. Число комбинаций в случае одновременного рассмотрения одного, двух и трех слоев составляет 3, 9 и 27 соответственно. Поскольку расчет с использованием одного слоя приводит к возникновению нефизичных осцилляций в рассчитанном распределении плотности ДН, а использование трех и более слоев, хотя и повышает незначительно точность вычислений, требует существенно больших трудозатрат [10], в данной работе, как наиболее оптимальное, использовалось одновременное рассмотрение двух слоев.

После определения комбинации подкорректированных значений „плотности ДН“ для слоев $i$ и $i+1$, первое 
из которых представляет собой искомую величину $\rho_{i}^{1}$, повторяем вышеописанную процедуру для следующей пары слоев $i+1$ и $i+2$. При этом, в качестве начального значения для $\rho_{i+1}$ выбираем подкорректированное значение ,Плотности ДН“ для слоя $i+1$, полученное на предыдущем шаге. Данная процедура повторяется до тех пор, пока не будут получены первые итерационные значения для всех подслоев. Следует отметить, что в качестве стартового значения поправки $\Delta \rho$ выбирается величина, превышающая значение максимально возможной плотности ДН в исследуемой структуре, соответствующей случаю полной релаксации в ней упругих напряжений (в настоящей работе $10^{12} \mathrm{~cm}^{-2}$ ).

На следующем этапе расчета величина поправки $\Delta \rho$ уменьшается на $\Delta \rho_{\text {step }}$ (например, на $10 \%$ от исходного значения) и процесс повторяется снова для каждого подслоя, пока не будут получены искомые значения $\rho_{i}^{2}$, $\rho_{i}^{3}$ и т.д. для всех подслоев. Процесс расчета останавливается по достижении требуемой точности определения плотности ДН $\Delta \rho^{\mathrm{f}}\left(50 \mathrm{~cm}^{-2}\right)$.

\section{3. Результаты и обсуждение}

Используя данный численный метод, мы провели расчет равновесного распределения плотности ДН и упругих напряжений вдоль направления эпитаксиального роста для различных конструкций МБС $\mathrm{In}_{x} \mathrm{Al}_{1-x} \mathrm{As}$, как традиционных, наиболее часто используемых в литературе: со ступенчатым, линейным или кусочнолинейным градиентом состава в направлении роста МБС $x(z)$, так и для получившего в последнее время развитие нелинейного МБС с корневой зависимостью $x(z)=x_{i}+\left(x_{\max }-x_{i}\right)(z / L)^{0.5}$, где $x_{i}$ и $x_{\max }$ - содержание In в начальной и конечной областях МБС. На рис. 1 представлены профили распределения плотности ДН по глубине $\rho(z)$ для перечисленных МБС, при этом толщина слоя, а также диапазон изменения состава твердого раствора выбирались одинаковыми для всех конструкций МБС $(L=1435 \mathrm{~nm}, x=0.05-0.87)$. В качестве ступенчатого МБС рассматривался слой, состоящий из 12 равных участков, на границах которых содержание In изменялось на одинаковую величину. Кусочно-линейный МБС состоял из двух слоев с линейным градиентом состава, величина которого уменьшалась в 1.5 раза при переходе от нижнего слоя к верхнему при $x=0.6$, по аналогии с работой [15]. Несмотря на сильное различие в характере представленных распределений, в верхней части каждой конструкции МБС присутствует область, где $\rho=0$, которая соответствует экспериментально наблюдаемой т. н. бездислокационной области.

Как видно из рис. 1, распределение $\rho(z)$ для ступенчатого МБС (кривая 1) представляет собой чередование узких пиков, соответствующих областям, где происходит скачкообразное изменение состава, сопровождаемое образованием ДН, и участков, в которых ДН полностью отсутствуют, при этом последний из

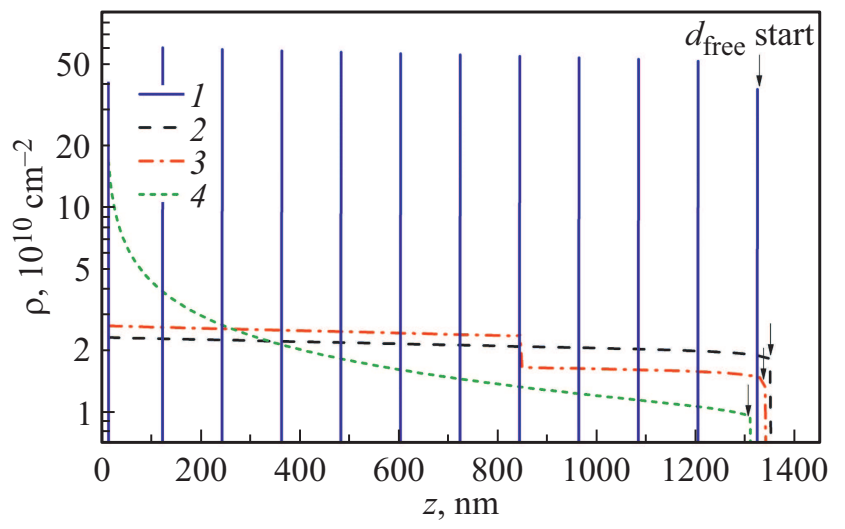

Рис. 1. Расчетные координатные зависимости плотности ДН вдоль направления роста, полученные для МБС $\operatorname{In}_{x} \mathrm{Al}_{1-x} \mathrm{As}$ $(L=1435 \mathrm{~nm}, x=0.05-0.87)$ с разным профилем изменения состава: 1) ступенчатый, 2) линейный, 3) кусочно-линейный, 4) корневой.

них выполняет роль области $d_{\text {free. }}$ Также необходимо отметить, что для данного типа МБС величина пиковых значений $\rho$ слабо изменяется по толщине структуры и в верхней части МБС, непосредственно прилегающей к области $d_{\text {free, }}$ достигает значения $\rho_{0} \sim 4 \cdot 10^{11} \mathrm{~cm}^{-2}$. В случае МБС с линейным профилем изменения состава ДН практически однородно распределены по толщине (кривая 2) и их плотность слабо уменьшается по мере роста МБС, достигая в верхней его части значения $\rho_{0} \sim 2 \cdot 10^{10} \mathrm{~cm}^{-2}$, более чем на порядок величины меньшего, чем в случае ступенчатого МБС. При анализе кусочно-линейного МБС получаем близкое по характеру с предыдущей конструкцией распределение плотности ДН (кривая 3), за исключением скачкообразного изменения $\rho$ в точке уменьшения градиента состава $(x=0.6, z=845 \mathrm{~nm})$, которое позволяет достигнуть незначительного спада $\rho$ в верхней части кусочнолинейного МБС $\left(\rho_{0} \sim 1.5 \cdot 10^{10} \mathrm{~cm}^{-2}\right)$ по сравнению с линейным. В случае корневого МБС профиль распределения $\rho(z)$ значительно отличается от остальных конструкций МБС, что отражается в нелинейном уменьшении плотности ДН более, чем на порядок величины по мере роста слоя, которая достигает значения $\rho_{0} \sim 9 \cdot 10^{9} \mathrm{~cm}^{-2}$ в верхней части МБС. Как видно из рис. 1, профили распределения $\rho(z)$ для разных конструкций сильно отличаются между собой не только характером, но и значением плотности ДН в области, непосредственно прилегающей к участку $d_{\text {free }}$, при этом минимальное значение $\rho_{0}$ достигается в случае корневого МБС. Также данный МБС характеризуется наибольшим значением $d_{\text {free }}=125 \mathrm{~nm}$, тогда как для ступенчатого, линейного и кусочно-линейного МБС данная величина составляет 115, 85 и $95 \mathrm{~nm}$ соответственно. Необходимо отметить, что полученные особенности распределения плотности ДН находят свое отражение и в эксперименте. В работе [16] методом ПЭМ были 


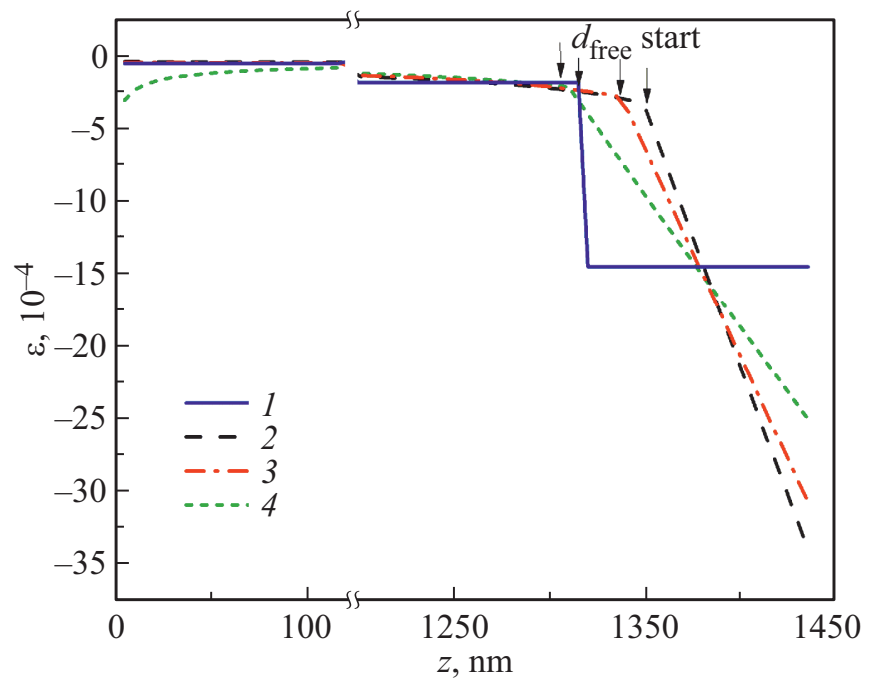

Рис. 2. Расчетные координатные зависимости величины упругих напряжений вдоль направления роста, полученные для МБС $\operatorname{In}_{x} \mathrm{Al}_{1-x}$ As $(L=1435 \mathrm{~nm}, x=0.05-0.87)$ разной конструкции: 1) ступенчатый, 2) линейный, 3) кусочно-линейный, 4) корневой.

проведены подробные исследования поперечных сечений образцов, содержащих МБС с корневым и кусочнолинейным профилем изменения состава, причем толщины слоев и диапазон изменения содержания In в них были близки по величине к рассматриваемым в данной работе параметрам. Полученные данные ПЭМ свидетельствуют о том, что в МБС с кусочно-линейным профилем изменения состава ДН действительно достаточно однородно распределены по глубине слоя вплоть до области $d_{\text {free }}$ в то время как в случае корневого МБС, величина $\rho$ значительно снижается вдоль направления роста. Следует также отметить, что экспериментально наблюдаемое значительное увеличение толщины $d_{\text {free }}$ в корневом МБС $(290 \mathrm{~nm})$ по сравнению с кусочнолинейным (180 nm) [16] достаточно хорошо согласуется с данными наших расчетов, показывающих соответствующее возрастание величины $d_{\text {free }}$ более чем на $30 \%$.

Результаты расчета профилей распределения упругих напряжений по глубине $\varepsilon(z)$ для МБС с различными типами градиента состава представлены на рис. 2. Следует отметить, что в отличие от зависимостей $\rho(z)$, которые сильно различаются между собой, зависимости $\varepsilon(z)$ для всех рассматриваемых типов конструкций МБС имеют схожий вид. Отрицательный знак величины $\varepsilon$ свидетельствует о том, что во всех данных МБС присутствуют только напряжения сжатия. При этом, практически по всей толщине слоя, за исключением области $d_{\text {free }}$, упругие напряжения достаточно малы и слабо изменяются в диапазоне от $(0.5-3) \cdot 10^{-4}$. В области свободной от ДН упругие напряжения резко возрастают, причем в случае ступенчатого МБС, они постоянны по толщине $d_{\text {free }}$ и составляют величину $\sim 1.5 \cdot 10^{-3}$, в то время как для МБС с линейными и корневым профилями изменения состава упругие напряжения линейно возрастают с толщиной, достигая в приповерхностной области МБС значений $\sim 3.5 \cdot 10^{-3}, \sim 3.1 \cdot 10^{-3}$ и $\sim 2.5 \cdot 10^{-3}$ для линейного, кусочно-линейного и корневого МБС соответственно. Несмотря на указанные различия, все слои характеризуются близкими значениями интегральной величины $\int_{L-d_{\text {free }}}^{L} \varepsilon d z$. Таким образом, между разными конструкциями МБС нет больших различий с точки зрения компенсации остаточных напряжений при последующем выращивании на них гетероструктур.

Проведенный анализ показывает, что МБС с корневым профилем изменения состава является наилучшей конструкцией буферного слоя, в которой основная плотность ДН сконцентрирована в нижней части МБС, более близкой к подложке GaAs. Отметим, что такая конфигурация ДН должна приводить к снижению плотности ПД, что и наблюдалось нами ранее экспериментально [16].

Рассмотрим далее, как будет изменяться распределение плотности ДН в этой конструкции МБС в зависимости от величины максимального содержания In и толщины слоя. На рис. 3 представлены профили распределения плотности ДН по глубине $\rho(z)$, полученные для корневого МБС с разной величиной $x_{\max }$ в предположении, что во всех случаях изменение состава с толщиной $x(z)$ происходит по одному закону (вставка на рис. 3). Как видно из рис. 3, достаточно сильные изменения величины максимального содержания In в корневом МБС от 75 до $87 \%$ практически не влияют на распределение плотности ДН по толщине, приводя лишь к незначительному снижению величины $\rho_{0}$ в верхней части МБС c $1.1 \cdot 10^{10}$ до $9 \cdot 10^{9} \mathrm{~cm}^{-2}$, а также увеличению толщины

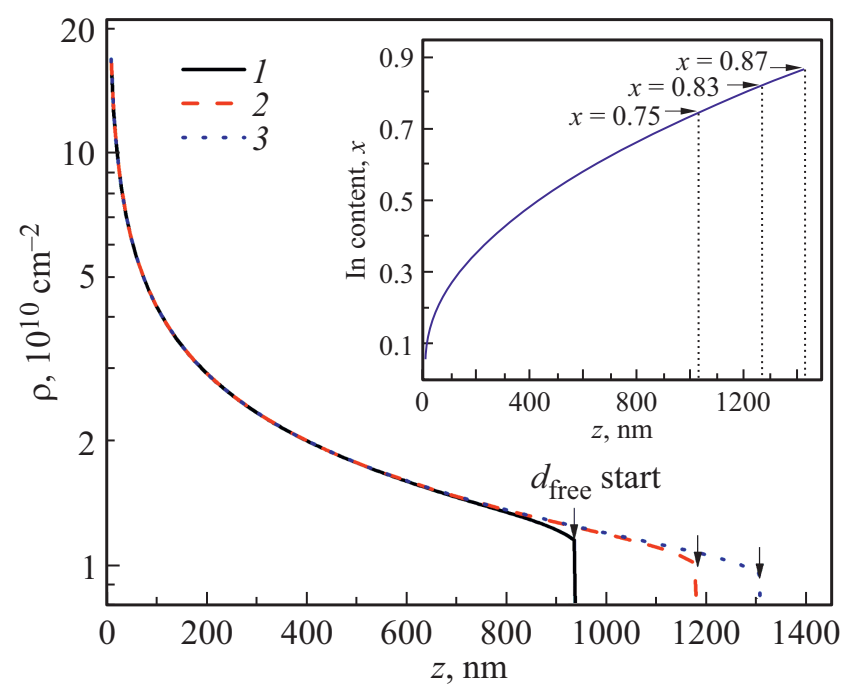

Рис. 3. Расчетные координатные зависимости плотности ДН вдоль направления роста, полученные для МБС $\operatorname{In}_{x} \mathrm{Al}_{1-x} \mathrm{As}$ с одним корневым профилем изменения состава при разных значениях параметров $x_{\max }$ и $L:$ 1) 0.75 и $\left.1050 \mathrm{~nm}, 2\right) 0.83$ и $1300 \mathrm{~nm}, 3) 0.87$ и $1435 \mathrm{~nm}$. На вставке приведен профиль изменения содержания In по толщине слоя, использованный для данных расчетов. 


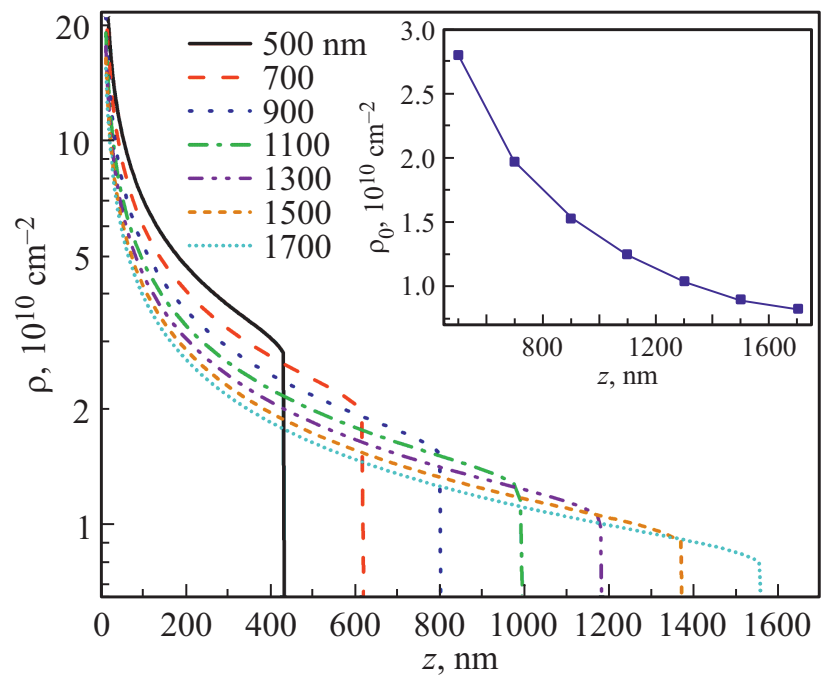

Рис. 4. Расчетные координатные зависимости плотности ДН вдоль направления роста, полученные для корневого МБС $\operatorname{In}_{x} \mathrm{Al}_{1-x} \mathrm{As}(x=0.05-0.87)$ разной толщины $(L=500-1700 \mathrm{~nm})$. На вставке приведена зависимость $\rho_{0}(L)$.

области $d_{\text {free }}$ от 110 до $125 \mathrm{~nm}$. Следует отметить, что наблюдаемые изменения $\rho_{0}$ и $d_{\text {free }}$ обусловлены скорее не столько изменением состава, сколько изменением толщины МБС. Это подтверждается расчетами зависимости $\rho(z)$ для корневого МБС с различной толщиной слоя, но фиксированным диапазоном изменения состава, результаты которых приведены на рис. 4.

Как видно из рис. 4, при всех, даже относительно малых толщинах распределение плотности ДН в корневом МБС носит неоднородный характер. Несмотря на то, что величина плотности ДН в верхней части МБС, непосредственно примыкающей к области $d_{\text {free, }}$, нелинейно зависит от толщины слоя (см. зависимость $\rho_{0}(L)$ на вставке рис. 4), значение $\rho_{0}$ даже при малой $L=500 \mathrm{~nm}$ не превышает $3 \cdot 10^{10} \mathrm{~cm}^{-2}$. Таким образом, можно предположить, что корневой МБС с меньшей в 2 раза толщиной, по сравнению с теми $(1000-1500 \mathrm{~nm})$, что обычно используются в традиционных конструкциях МБС, не будет уступать им по кристаллографическому качеству и плотности ПД. Данное обстоятельство, несомненно, является важным при практической реализации метаморфных гетероструктур.

Следует отметить, что используемый в настоящей работе упрощенный подход для расчета плотности ДН и остаточных упругих напряжений в МБС не учитывает эффекта взаимодействия дислокаций между собой. Данный эффект может оказывать существенное влияние как на распределение плотности ДН и ПД, так и на снижение их величины. Тем не менее, такие расчеты, как было показано, позволяют правильно предсказать особенности в распределении плотности ДН и упругих напряжений в разных типах МБС и могут быть эффективно использованы при конструировании приборных метаморфных гетероструктур.

\section{4. Заключение}

Проведен расчет равновесных распределений плотности ДН $\rho(z)$ и упругих напряжений $\varepsilon(z)$ вдоль направления эпитаксиального роста для МБС $\operatorname{In}_{x} \mathrm{Al}_{1-x} \mathrm{As}$ с высоким содержанием In (до 87 мол.\%) и разным типом градиента состава: ступенчатым, линейным (кусочно-линейным) и корневым. Показано, что данные конструкции МБС имеют сильное различие между собой в характере распределения $\rho(z)$, в то время как зависимости $\varepsilon(z)$ имеют схожий вид, отличаясь

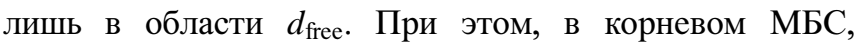
в отличие от традиционных (ступенчатого и линейного) с практически однородным распределением ДН, зависимость $\rho(z)$ строго нелинейна, причем основная часть ДН сконцентрирована в нижней части МБС вблизи гетерограницы с подложкой GaAs. Отметим, что указанные особенности наблюдались нами ранее экспериментально. Кроме того, установлено, что именно корневой МБС характеризуется наименьшим среди всех конструкций значением плотности ДН в верхней части слоя вплоть до $\rho_{0} \sim 9 \cdot 10^{9} \mathrm{~cm}^{-2}$ и наибольшей толщиной бездислокационной области $d_{\text {free }}=125 \mathrm{~nm}$. Следует отметить, что хотя зависимости $\varepsilon(z)$ отличаются для разных МБС в области $d_{\text {free }}$, интегральная величина остаточных напряжений в бездислокационной области для них практически одинакова, свидетельствуя, что между разными конструкциями МБС нет больших различий с точки зрения компенсации остаточных напряжений при последующем выращивании на МБС гетероструктур. Использованный для расчетов в настоящей работе численный метод не учитывает важный эффект взаимодействия дислокаций между собой, приводящий к снижению значений абсолютной плотности ПД. Тем не менее, он позволил качественно воспроизвести все наблюдаемые экспериментально особенности распределения плотности ДН в различных МБС и установить характер распределения в них упругих напряжений. Таким образом, данный подход может быть эффективно использован при разработке оптимальных конструкций приборных метаморфных гетероструктур различного максимального состава и требуемой толщины.

\section{Финансирование работы}

Работа выполнена при частичной поддержке Российского фонда фундаментальных исследований (проект № 18-02-00950).

\section{Конфликт интересов}

Авторы заявляют об отсутствии конфликта интересов. 


\section{Список литературы}

[1] G.B. Galiev, I.S. Vasil'evskii, S.S. Pushkarev, E.A. Klimov, R.M. Imamov, P.A. Buffat, B. Dwir, E.I. Suvorov. J. Cryst. Growth 366, 55 (2013).

[2] W. Hafez, J. Lai, M. Feng. Electron. Lett. 39, 1447 (2003).

[3] G. Belenky, D. Wang, Y. Lin, D. Donetsky, G. Kipshidze, L. Shterengas, D. Westerfeld, W.L. Sarney, S. Svensso. Appl. Phys. Lett. 102, 111108 (2013).

[4] S.V. Ivanov, M.Yu. Chernov, V.A. Solov'ev, P.N. Brunkov, D.D. Firsov, O.S. Komkov. Prog. Cryst. Growth Charact. Mater. 65, 20 (2019).

[5] V.A. Kulbachinskii, L.N. Oveshnikov, R.A. Lunin, N.A. Yuzeeva, G.B. Galiev, E.A. Klimov, S.S. Pushkarev, P.P. Maltsev. Semicond. 49, 921 (2015).

[6] D.J. Dunstan, P. Kidd, L.K. Howard, R.H. Dixon. Appl. Phys. Lett. 59, 3390 (1991).

[7] D.J. Dunstan. J. Mater. Sci. Mater. Electron. 8, 337 (1997).

[8] H. Choi, Y. Jeong, J. Cho, M.H. Jeon. J. Cryst. Growth 311, 1091 (2009).

[9] M.Yu. Chernov, V.A. Solov'ev, O.S. Komkov, D.D. Firsov, B.Ya. Meltser, M.A. Yagovkina, M.V. Baidakova, P.S. Kop'ev, S.V. Ivanov. Appl. Phys. Express 10, 121201 (2017).

[10] B. Bertoli, E.N. Suarez, J.E. Ayers, F.C. Jain. J. Appl. Phys. 106, 073519 (2009).

[11] S. Adachi. Properties of Semiconductor Alloys: Group-IV, IIIV and II-VI Semiconductors. Wiley-Blackwell (2009). 413 p.

[12] E.A. Fitzgerald. Mater. Sci. Rep. 7, 87 (1991).

[13] J. Zou, D.J.H. Cockayne, B.F. Usher. J. Appl. Phys. 73, 619 (1993).

[14] I.N. Trunkin, M.Yu. Presniakov, A.L. Vasiliev. Crystallography Rep. 62, 265 (2017).

[15] F. Capotondi, G. Biasiol, D. Ercolani, V. Grillo, E. Carlino, F. Romanato, L. Sorba. Thin Solid Films 484, 400 (2005).

[16] В.А. Соловьев, М.Ю. Чернов, А.А. Ситникова, П.Н. Брунков, Б.Я. Мельцер, С.В. Иванов. ФТП 52, 127 (2018).

Редактор Ю.Э. Китаев 